\title{
A PÓS-GRADUAÇÃO E A SÍNDROME DE BURNOUT: ESTUDO COM ALUNOS DE MESTRADO EM ADMINISTRAÇÃO
}

\author{
A POST-GRADUATE AND BURNOUT SYNDROME: A STUDY OF STUDENTS \\ WITH MASTERS' DEGREE IN ADMINISTRATION
}

\author{
Rayssa Soares de Souza \\ Universidade Federal do Rio Grande do Norte
}

Renata Paula Costa Trigueiro

Universidade Federal do Rio Grande do Norte

Tatiane Nunes Viana de Almeida

Universidade Federal do Rio Grande do Norte

\section{José Arimátes de Oliveira}

Universidade Federal do Rio Grande do Norte

\section{RESUMO}

Burnout é uma síndrome psicológica considerada grave que pode afetar as várias dimensões da vida do sujeito: a vida pessoal, a sua auto avaliação e suas relações interpessoais. Neste sentido, o objeto de estudo dessa pesquisa foram estudantes do Programa de Pós-Graduação em Administração da Universidade Federal do Rio Grande do Norte. Esta pesquisa objetivou identificar as três dimensões da síndrome e estabelecer a correlação entre os fatores da síndrome e as variáveis sócio-demográficas e acadêmicas. Os dados foram coletados através do MBI-SS e analisados com o auxílio do SPSS 15.0. As variáveis foram enquadradas diferentemente dos resultados encontrados pela versão original do instrumento. E constataram-se relações significativas entre as variáveis sócio-demográficas e acadêmicas com as dimensões da síndrome de burnout. Por fim, salienta-se a dificuldade em classificar os sujeitos nos níveis de baixo, moderado e alto, apontando para a necessidade de aprofundamento dos resultados obtidos. Assim, sugere-se a realização de novos estudos, com outras variáveis e categorias estudantis.

Palavras-chave: Burnout. Estudantes. Mestrado. MBI-SS.

\section{ABSTRACT}

Burnout is considered a serious psychological syndrome that can affect the various dimensions of the life: personal life, your self assessment and their interpersonal relationships. In this sense, the object of this research was students of the master's degree at the Universidade Federal do Rio Grande do Norte. This research aimed to identify the three dimensions of the syndrome and establish the correlation between the factors of the syndrome and socio-demographic and academic variables. Data were collected through the MBI-SS and analyzed with the aid of SPSS 15.0. The variables of the MBI-SS were framed differently from the results reported on the original version of the instrument. And it was found significant relationships between socio-demographic and academic variables with the dimensions of the burnout syndrome. Finally, salient the difficulty in classifying the subjects in the levels of low, moderate and high, pointing to the necessity of deepening the results. Thus, it is suggested to carry out further studies with other variables and student categories.

Keywords: Burnout. Students. Master's degree. MBI-SS. 


\section{INTRODUÇÃO}

A dinâmica do mundo moderno elevou os níveis de estresse, tornando-o inevitável aos indivíduos na maioria das organizações. Considerado como um fenômeno complexo, o estresse tem sido definido sob várias óticas, de acordo com a área de conhecimento e com o contexto em que está sendo enfocado. A multiplicidade dos agentes potencialmente estressores fez aumentar consideravelmente o seu nível, ocasionando a síndrome de burnout, uma das patologias que surgiu na década de 70, em decorrência da percepção de más condições de trabalho. Uma síndrome psicológica que se caracteriza por reações de exaustão física e emocional, dificuldades nos relacionamentos com as pessoas, colegas e superiores e com o próprio trabalho, bem como sentimentos de falta de realização pessoal, eficácia e produtividade (MASLACH, 1997), o que afeta gravemente os indivíduos e pode gerar altos custos para a organização.

Inicialmente os estudos sobre burnout estavam relacionados diretamente com a dimensão do trabalho, entretanto, com a evolução das pesquisas sobre a temática, foi verificado que a síndrome pode ser identificada em outras esferas da vida, como na relação entre pais e filhos, entre membros de um casal, em estudantes, enfim, em outras relações que não envolvam necessariamente o trabalho remunerado.

0 ambiente acadêmico, especialmente o da pós-graduação, é caracterizado por estímulos múltiplos, competitividade, cumprimento de metas que estabelece para os alunos e professores uma rotina de cobranças, de obrigações, tensões e exigências, as mais diversas. Diante do exposto, acredita-se ser relevante a investigação da síndrome de burnout em estudantes de pós-graduação, bem como sua associação com as variáveis sócio-demográficas e acadêmicas.

De acordo com Dimsdale e Baum (apud LIPP, 2000), o nível de tensão experienciado por uma pessoa, principalmente se for persistente e ultrapassar sua resistência, pode interferir na sua qualidade de vida, na saúde física e mental e na sua produtividade. Portanto, o burnout pode trazer graves prejuízos pessoais e organizacionais.

Neste estudo buscou-se identificar as três dimensões da síndrome (exaustão, cinismo e realização pessoal) em uma amostra de 45 alunos do Programa de Pós-Graduação em Administração da Universidade Federal do Rio Grande do Norte, bem como estabelecer a correlação entre os fatores da síndrome e as variáveis sócio-demográficas e acadêmicas.

\section{BURNOUT}

0 termo burnout é uma expressão originada da gíria inglesa, que foi utilizada para referir-se à pessoa que se acabou pelo excesso de uso de drogas. 0 termo pode ser traduzido como "queimar-se", "estar acabado" (FRANÇA, 1987). Para Benevides-Pereira (2002, p. 21) a expressão foi significada como o estado daquele "que chegou ao limite e por falta de energia, não tem mais condições de desempenho físico e mental".

Atribui-se a Freudenberger (apud TAMAYO, 1997) a origem do termo burnout como problema de saúde ocasionado pelo trabalho, no entanto, Schaufeli e Ezmann (apud BENEVIDES-PEREIRA, 2002, p. 21) afirmam que "Brandley já o havia utilizado em 1969, propondo uma nova estrutura organizacional a fim de conter o fenômeno psicológico que acomete trabalhadores assistenciais". Entretanto, a síndrome de burnout, só tenha despertado interesse científico após os estudos de Freudenberger e Maslach.

Os estudos, desde então, tem avançado e tomado novas dimensões. Inicialmente acreditava-se que a síndrome acometia apenas pessoas que tinham ocupações relacionadas a cuidados pessoais e serviços assistenciais como atendimento à saúde, saúde mental, assistência social, sistema judiciário penal, profissões religiosas, aconselhamento e ensino (MASLACH, 2007), onde o foco principal seria o de auxiliar pessoas necessitadas e que era uma síndrome que estava relacionada apenas ao ambiente 
laboral. No entanto, Maslach e Leiter (1997) afirmam que em virtude das mudanças ocorridas no mundo do trabalho e as exigências de um serviço 'personalizado' ao cliente, cada vez mais trabalhadores em diversas profissões têm sido acometidos pela síndrome.

Com a evolução dos estudos na área, foi identificado a existência do burnout em outras formas de relacionamento, conforme afirma Maslach (2007, p. 43):

Embora o Burnout tenha sido identificado principalmente como um fenômeno do mundo do trabalho, a importância do contexto social e das relações interpessoais para o Burnout sugere que ele pode ser relevante a outras esferas da vida.

Já existindo pesquisas sobre burnout em relação a pais e filhos e na relação entre os membros de um casal (PROCACCINI; KIEFABER, 1983; PINES, 1996 apud MASLACH, 2007), o que demonstra o avanço nos estudos.

Para Maslach (2007), burnout é uma grave síndrome psicológica, decorrente da exposição prolongada de um indivíduo a um estressor interpessoal crônico, que possui três dimensões: a exaustão emocional, o componente individual da síndrome, que é o sentimento de esgotamento físico e emocional; a despersonalização, o componente interpessoal, comumente a última dimensão a ser afetada, que é quando o indivíduo começa a apresentar problemas para a empresa e para seu grupo de trabalho, com suas atitudes de ironia, cinismo, hostilidade e por fim, a falta de realização pessoal, o componente da auto-avaliação, onde o indivíduo não se sente mais capaz, duvida da sua capacidade de realização e por vezes apresenta ímpeto de abandonar sua ocupação.

Burnout seria, então, um processo de erosão psicológica decorrente da exposição crônica a estressores ocupacionais contínuos que tem efeito cumulativo e pouco a pouco vai minando as defesas dos indivíduos (MASLACH, 2007). A autora diz ainda que, as organizações precisam estar atentas a esse processo de erosão, pois este pode ter custos altos, tanto para as organizações, quanto para os funcionários, além de predizer piores desempenhos, insatisfações com o emprego, baixo comprometimento organizacional, absenteísmo, problemas de relacionamentos em geral e problemas de saúde.

\section{BURNOUT EM ESTUDANTES DE PÓS-GRADUAÇÃO}

A entrada na pós-graduação, especificamente a strictu sensu, exige do indivíduo um grande esforço de adaptação, tendo em vista que o estudante terá que desenvolver, dentre outras atividades de alto desempenho, o papel de pesquisador.

Conforme expõem Voltarelli (2002) e Santos e Alves Júnior (2007), a produtividade acadêmica pode ser um estressor para os pesquisadores nacionais, tendo em vista que o imperativo de dedicação exclusiva, por muitas vezes sem um apoio financeiro, a ênfase em publicação - tanto no âmbito nacional quanto no internacional - e a concorrência desleal devido à falta de estabilidade profissional pode gerar um estado de esgotamento mental, prejudicando, dessa forma, a saúde do indivíduo. Complementando, Duque, Brondani e Luna (2005) salientam que além da carência de recursos e do ambiente competitivo, lidar com a pressão dos prazos, conciliar as inúmeras atividades acadêmicas e as incertezas em relação ao futuro profissional também podem ser considerados fatores estressantes.

Nos programas de pós-graduação no Brasil - mestrado e doutorado - são realizados seminários com o objetivo de induzir os estudantes à realização de pesquisa, aprimorando a escrita, a leitura do caso pesquisado e a produção do trabalho final (Trzesniak, 2004). No entanto, devido a inúmeros motivos dentre os quais a falta de dedicação devido à sobrecarga de atividades dentro e fora da pós-graduação, Trzesniak (2004) afirma que a experiência tem apontado que é complicado manter tanto a realização de seminários quanto a confecção das dissertações e teses em um patamar satisfatório para a coordenação dos programas, o que pode suscitar sintomas do distresse (estresse negativo) para o estudante. Quando esse estressor persiste e é considerado crônico para os sujeitos, estes podem 
desenvolver a síndrome de burnout.

Inicialmente, o conceito de burnout era relacionado aos profissionais de serviços sociais e de saúde. No entanto, o maior interesse no assunto tem feito com que o campo de estudo fosse ampliado. 0 conceito de burnout para estudantes - uma atividade pré-profissional - foi proposto com rigidez e base empírica por Schaufeli, Salanova, González-Romá e Bakker (2002). Para Cushway (1992 apud CARLOTTO; CÂMARA, 2006) a síndrome de burnout pode começar na fase acadêmica e estudos têm apontado que o burnout pode ter início no período de formação e perdurar durante a vida profissional.

De acordo com Carlotto e Câmara (2006), o burnout nos estudantes também compreende três dimensões: exaustão - sentimento de estar exausto devido às exigências dos estudos; cinismo desenvolvimento de uma atitude cínica e/ou distanciada em relação aos estudos; e, inrealização pessoal (inversão da dimensão "realização pessoal") - percepção de estar sendo incompetente como estudante.

Um estudo com estudantes de diversos cursos, desenvolvido por Agut, Grau e Beas (2002 apud BORGES; CARLOTTO, 2004), encontrou nível de burnout moderados nas três dimensões. Todavia, os estudantes dos cursos da área de ciência humanas apresentaram um maior nível de esgotamento mental. De uma forma geral, a síndrome de burnout pode levar a diminuição progressiva do idealismo, além de prejudicar o desempenho funcional e a saúde física e mental do indivíduo (VOLTARELLI, 2002).

Diante do exposto, acredita-se ser relevante a investigação da síndrome de burnout em estudantes de pós-graduação em Administração, bem como sua associação com as variáveis sócio-demográficas e acadêmicas. Através dos resultados obtidos, busca-se detectar índices significativos que possam afetar o êxito do estudante e em última instância, a desistência do curso, possibilitando, dessa forma, intervenções preventivas, já que Maslach (2007) considera ser o burnout não um problema dos indivíduos, mas sim das Organizações.

\section{MÉTODO}

Desenvolvida com o objetivo de verificar a incidência da síndrome de burnout em estudantes de PósGraduação em Administração, bem como a identificar a existência de relação entre as dimensões do burnout e as variáveis sócio-demográficas e acadêmicas, a presente pesquisa é do tipo exploratório e descritivo, de natureza quantitativa.

A abordagem exploratória "visa prover o pesquisador de maior conhecimento sobre o tema ou problema em perspectiva" (MATTAR, 1996, p.18), enquanto que a descritiva tem como finalidade expor as peculiaridades de determinada população (GIL, 1999).

A população pesquisada foi constituída pelos estudantes do Programa de Pós-Graduação em Administração da Universidade Federal do Rio Grande do Norte. A intenção da pesquisa era realizar um estudo censitário, o que não foi possível, portanto, a amostra foi não-probabilística por conveniência, tendo em vista que os pesquisados foram selecionados por critérios subjetivos das pesquisadoras, de acordo com os objetivos de estudo e com a disponibilidade dos indivíduos em responder o questionário (SAMARA; BARROS, 2002). Salienta-se que tal amostra traz o inconveniente do estudo não poder ser generalizado a todos os estudantes da população pesquisada.

A coleta de dados foi realizada no período de 16 a 20 de junho de 2008. Tal estudo contou com a participação de 45 alunos dos 48 ativos das turmas 29 e 30 do Mestrado em Administração da UFRN, com a ressalva de três sujeitos que se negaram responder a pesquisa. 0 questionário foi entregue a todos os mestrandos, respondidos por livre consentimento e devolvidos as pesquisadoras.

O instrumento é composto de duas partes. A primeira, destinada a identificação do perfil sóciodemográfico e acadêmico dos respondentes e a segunda, a incidência do burnout, através do Maslach 
Burnout Inventory - Student Survey (MBI-SS), adaptado, com escala do tipo Likert de cinco pontos, referindo-se a frequência em que o sujeito diz experimentar o conteúdo sugerido pelo item.

O MBI-SS é uma adaptação do Maslach Burnout Inventory - General Survey (MBIGS) para estudantes, realizado por Schaufeli e colaboradores (2002). Tal instrumento contém 15 itens que se subdividem em três dimensões: exaustão (cinco itens), cinismo (quatro itens) e realização pessoal (seis itens) (tabela 1). Maslach (1986) considera a síndrome uma variável contínua que apresenta níveis alto, moderado e baixo, dependendo da frequência com que o indivíduo experimenta os sentimentos relacionados com cada dimensão (ou fator).

\section{Tabela 1: Dimensões do burnout - MBI-SS}

\begin{tabular}{|c|c|}
\hline Dimensão & Itens - MBI-SS \\
\hline Exaustão & $\begin{array}{l}\text { Item 1- Sinto-me emocionalmente esgotado pelos meus estudos. } \\
\text { Item 4- Sinto-me esgotado no fim de um dia em que tenho aula. } \\
\text { Item 6- Sinto-me cansado quando me levanto para enfrentar outro dia de aula. } \\
\text { Item 8- Estudar e freqüentar as aulas são, para mim, um grande esforço. } \\
\text { Item 12- Sinto-me consumido pelos meus estudos. }\end{array}$ \\
\hline Cinismo & $\begin{array}{l}\text { Item 2- Eu questiono o sentido e a importância de meus estudos. } \\
\text { Item 9- Tenho me tornado menos interessado nos estudos desde que entrei nesta universidade. } \\
\text { Item 10- Tenho me tornado menos interessado nos meus estudos. } \\
\text { Item 14- Tenho estado mais descrente do meu potencial e da utilidade dos meus estudos. }\end{array}$ \\
\hline $\begin{array}{c}\text { Realização } \\
\text { pessoal }\end{array}$ & $\begin{array}{l}\text { Item 3- Tenho aprendido muitas coisas interessantes no decorrer dos meus estudos. } \\
\text { Item 5- Durante as aulas, sinto-me confiante: realizo as tarefas de forma eficaz. } \\
\text { Item 7- Sinto me estimulado quando concluo com êxito a minha meta de estudos. } \\
\text { Item 11- Considero-me um bom estudante. } \\
\text { Item 13- Posso resolver calmamente os problemas que surgem nos meus estudos. } \\
\text { Item 15- Acredito que eu seja eficaz na contribuição das aulas que frequiento. }\end{array}$ \\
\hline
\end{tabular}

Fonte: Schaufeli e colaboradores (2002)

Um alto nível de burnout está relacionado com a apresentação concomitante de altos escores nos fatores de exaustão e cinismo e baixos escores na dimensão realização pessoal, enquanto que um nível moderado refere-se a presença de escores médios nas três subescalas e um nível baixo está relacionado a existência de baixos escores nos fatores exaustão e cinismo e altos escores no fator realização pessoal.

Salienta-se que Tamayo (1997) considerou em seus estudos os seguintes valores de referência: para alto, valores superiores ao percentil 67; moderado, os escores entre os percentis 33 e 67; e, baixos, os valores inferiores ao percentil 33.

Os dados obtidos na coleta de dados foram analisados através do SPSS 15.0 for Windows. Inicialmente foram realizados os testes de suposição da análise multivariada: normalidade, homoscedasticidade e linearidade. Após essa primeira etapa, foram submetidos a análise descritiva - média e desvio padrão - e inferencial - correlação de Pearson e análise por componentes principais, para identificar as três dimensões da síndrome de burnout e as associações entre a síndrome (variável dependente) e as variáveis sócio-demográficas e acadêmicas (variáveis independentes).

\section{ANÁLISE E DISCUSSÃO DOS RESULTADOS}

A amostra estudada é composta por 45 alunos do Mestrado em Administração da UFRN, sendo 26 $(57,8 \%)$ da 29 a turma e 19 alunos $(42,2 \%)$ da 30a turma. A referida amostra é composta por 24 indivíduos $(53,3 \%)$ do sexo feminino e $21(46,7 \%)$ do sexo masculino. Quanto à faixa etária, predominou a de 21 a 25 anos (35,6\%), seguida da de 26 a 30 anos (31,1\%), de mais de 40 anos com $20 \%$, de 31 a 35 anos com 8,9\% e de 36 a 40 com 4,4\%. A maior parte dos estudantes não tem filhos 
$(75,6 \%)$, mora com a família $(68,9 \%)$ e é solteira $(68,9 \%)$. Dentre os demais, $24,4 \%$ são casados, $4,4 \%$ vivem em união estável e $2,2 \%$ são viúvos. Quanto à realização de outras atividades, além do mestrado, 64,4\% disseram ter uma atividade remunerada e 35,6\% ter dedicação exclusiva ao Programa. Além disso, os pesquisados foram indagados sobre a realização de atividades de lazer e a satisfação com o curso: a maioria não pratica nenhuma atividade regular de lazer $(62,2 \%)$ e está satisfeito com a pós-graduação, seguidos por uma parcela muito satisfeita $(31,1 \%)$ e outra indiferente $(4,4 \%)$.

Após o cálculo das freqüências do perfil sócio-demográfico, foi verificado o Alfa de Crombach. De acordo com o SPSS, os coeficientes do Alfa de Cronbach não padronizado e padronizado são, respectivamente, 0,575 e 0,492, com base em 15 itens (variáveis). Esses dados demonstram que a escala não é consistente, considerando que a literatura recomenda a consistência acima de 0,7 (HAIR, 2005). De acordo com Tamayo (1997) esse baixo índice de consistência interna pode ser justificado a partir da influência da desejabilidade social na resposta do instrumento. Tal influência tem respaldo nas idéias Freudenberger (apud TAMAYO, 1997) que defende que os indivíduos com burnout acreditam em uma imagem idealizada de si mesmos, tendo, portanto, dificuldade para admitir possíveis falhas em qualquer das dimensões do seu trabalho.

No que se refere ao MBI-SS, as 15 variáveis do instrumento foram submetidas, inicialmente, à análise de componentes principais. Para determinar a adequação de tal análise foi utilizado o teste de KMO (Kaiser-Meyer-Olkin) e de Esfericidade de Bartlett de esfericidade, conforme apresenta a tabela 2.

Tabela 2: KMO e Teste de Esfericidade de Bartlett

\begin{tabular}{clr}
\hline \multicolumn{2}{c}{ Medida de Adequação da Amostra (KMO) } & $\mathbf{0 , 7 2 3}$ \\
\hline \multirow{3}{*}{ Teste de Barlett } & Chi-quadrado aproximado & 366,728 \\
& Grau de liberdade (df) & 105 \\
& Significância (sig.) & 0,000 \\
\hline
\end{tabular}

Fonte: Dados da pesquisa, 2008

De acordo com os dados gerados pelo SPSS, o teste de Esfericidade de Barlett apresentou significância de 0,001 , apontando para um nível de correlação não-nula apropriada para a amostra do estudo. Em relação ao KMO, a amostra apresentou um índice de 0,723, acima do valor mínimo de 0,50 aconselhável por Corrar, Paulo e Dias Filho (2007) . Logo, é possível ter consistência para prosseguir o uso da análise fatorial.

Foram extraídos três fatores que explicam $64,054 \%$ da variância total, sendo: o primeiro fator explica $28,510 \%$ da variância, o segundo $19,829 \%$ e terceiro $15,715 \%$. Foi utilizada a rotação ortogonal varimax e considerados somente os índices de carga fatorial acima de 0,50 (tabela 3). 
Tabela 3: Matriz dos Componentes Rotacionada a de cada dimensão

\begin{tabular}{|c|c|c|c|c|}
\hline \multirow{2}{*}{\multicolumn{2}{|c|}{ Item - MBI-SS }} & \multirow{2}{*}{\multicolumn{3}{|c|}{ Dimensão }} \\
\hline & & & & \\
\hline 1 & Sinto-me emocionalmente esootado pelos meus estudos. & 0828 & & \\
\hline 2 & Eu questiono o sentido e a importância de meus estudos. & 0,623 & & \\
\hline 4 & Sinto-me esgotado no fim de um dia em que tenho aula. & 0,756 & & \\
\hline 6 & $\begin{array}{l}\text { Sinto-me cansado quando me levanto para enfrentar outro dia de } \\
\text { aula. }\end{array}$ & 0,683 & & \\
\hline 8 & Estudar e freqüentar as aulas são, para mim, um grande esforço. & 0,587 & & \\
\hline 12 & Sinto-me consumido pelos meus estudos. & 0,857 & & \\
\hline 14 & $\begin{array}{l}\text { Tenho estado mais descrente do meu potencial e da utilidade dos } \\
\text { meus estudos. }\end{array}$ & 0,655 & & \\
\hline 5 & $\begin{array}{l}\text { Durante as aulas, sinto-me confiante: realizo as tarefas de forma } \\
\text { eficaz. }\end{array}$ & & 0,712 & \\
\hline 7 & $\begin{array}{l}\text { Sinto me estimulado quando concluo com êxito a minha meta de } \\
\text { estudos. }\end{array}$ & & 0,505 & \\
\hline 11 & Considero-me um bom estudante. & & 0,795 & \\
\hline 13 & $\begin{array}{l}\text { Posso resolver calmamente os problemas que surgem nos meus } \\
\text { estudos. }\end{array}$ & & 0,513 & \\
\hline 15 & Acredito que eu seja eficaz na contribuição das aulas que freqüento. & & 0,872 & \\
\hline 9 & $\begin{array}{l}\text { Tenho me tornado menos interessado nos estudos desde que entrei } \\
\text { nesta universidade. }\end{array}$ & & & 0,882 \\
\hline 10 & Tenho me tornado menos interessado nos meus estudos. & & & 0,870 \\
\hline & Alpha de Cronbach & 0,869 & 0,827 & 0,890 \\
\hline
\end{tabular}

Método de Extração: Análise de Componentes Principais.

Método de Rotação: Varimax com Normalização de Kaiser.

a: A rotação convergiu em sete interações.

Fonte: Dados de pesquisa, 2008

Os resultados confirmaram 14 itens distribuídos em três fatores, conceitualmente relacionados a exaustão (10 itens), realização pessoal (5 itens) e cinismo ( 2 itens). Para todos os fatores foi considerado o critério de carga fatorial > 0,50, conforme orienta Corrar, Paulo e Dias Filho (2007).

Com relação ao instrumento original, ocorreram as seguintes mudanças:

Fator 1 - Exaustão: Esse fator ficou composto por 7 itens, os mesmos 5 que compõem na versão original, além dos itens 2 (Eu questiono o sentido e a importância de meus estudos) e 14 (Tenho estado mais descrente do meu potencial e da utilidade dos meus estudos). No instrumento original, os itens supracitados compunham a dimensão do cinismo.

Fator 2 - Realização Pessoal: Esse fator ficou composto por 5 itens, os mesmos 5 que compõe -no na versão original, com exceção do item 3 (Tenho aprendido muitas coisas interessantes no decorrer dos meus estudos) que apresentou uma carga fatorial inferior a 0,50.

Fator 3 - Cinismo: No instrumento original, esse fator era composto por 4 itens. No entanto, no presente estudo esse fator ficou com 2 fatores, tendo em vista que os outros 2 ficaram no fator 1, de exaustão.

Diferentemente do que ocorreu quando foi analisada a consistência interna das 15 variáveis do instrumento original, ao examinar a consistência por dimensão obteve-se índices satisfatórios, tendo em vista que os fatores de exaustão $(0,869)$, de realização pessoal $(0,827)$ e de cinismo $(0,890)$ foram superiores a 0,7 conforme indica a literatura (HAIR, 2005). Logo, a partir dos resultados obtidos, podese concluir que cada uma das dimensões do MBI-SS tem sua confiabilidade interna dentro dos padrões 


\section{A pós-graduação e a síndrome de burnout: estudo com alunos de mestrado em Administração}

de aceitação.

Os resultados da correlação entre as dimensões do burnout e as variáveis sóciodemográficas, apresentaram as seguintes relações significativas ao nível de 5\%: na dimensão Exaustão - sexo, faixa etária e filhos; na dimensão Realização Pessoal - sexo, faixa etária e estado civil; e, na dimensão Cinismo - estado civil (tabela 4).

Tabela 3: Relação entre dimensões de burnout e variáveis sócio-demográficas

\begin{tabular}{|c|c|c|c|c|c|c|c|c|c|}
\hline \multirow[t]{2}{*}{$\overline{\text { Dimensões }}$} & \multicolumn{3}{|c|}{ Exaustão } & \multicolumn{3}{|c|}{ Realização Pessoal } & \multicolumn{3}{|c|}{ Cinismo } \\
\hline & $\mathbf{M}$ & SD & p & $\mathbf{M}$ & SD & p & $\mathbf{M}$ & SD & $\mathbf{p}$ \\
\hline \multicolumn{10}{|l|}{ Variáveis } \\
\hline \multicolumn{10}{|l|}{ Sexo } \\
\hline Feminino & 22,42 & 4,49 & & 16,42 & 2,55 & & 4,00 & 2,00 & \\
\hline Masculino & 18,48 & 7,41 & $0,03 *$ & 19,71 & 3,65 & $0,01 *$ & 3,62 & 2,22 & 0,54 \\
\hline \multicolumn{10}{|l|}{ Faixa Etária } \\
\hline De 21 a 25 & 23,81 & 3,41 & & 16,31 & 3,46 & & 4,13 & 2,19 & \\
\hline De 26 a 30 & 20,36 & 6,91 & & 18,71 & 3,20 & & 3,57 & 1,70 & \\
\hline Mais de 31 & 17,33 & 6,63 & $0,03 *$ & 19,00 & 3,36 & $0,02 *$ & 3,73 & 2,40 & 0,60 \\
\hline \multicolumn{10}{|l|}{ Estado Civil } \\
\hline Solteiro (a) & 21,61 & 5,64 & & 18,00 & 2,88 & & 3,55 & 1,95 & \\
\hline Casado (a) & 16,81 & 7,39 & & 19,27 & 3,90 & & 3,90 & 1,92 & \\
\hline Outro & 23,67 & 3,06 & 0,69 & 12,67 & 4,16 & $0,03^{*}$ & 6,33 & 3,21 & $0,03 *$ \\
\hline \multicolumn{10}{|l|}{ Filhos } \\
\hline Sim & 15,27 & 4,73 & & 18,91 & 3,81 & & 3,27 & 1,68 & \\
\hline Não & 22,29 & 5,78 & $0,001 *$ & 17,65 & 3,39 & 0,30 & 4,00 & 2,20 & 0,32 \\
\hline \multicolumn{10}{|l|}{ Moradia } \\
\hline Família & 21,10 & 6,56 & & 17,98 & 3,08 & & 3,77 & 1,73 & \\
\hline A migos & 18,85 & 7,27 & & 16,29 & 5,47 & & 3,71 & 2,87 & \\
\hline Sozinho & 20,00 & 4,00 & 0,52 & 19,57 & 2,44 & 0,53 & 4,14 & 2,97 & 0,72 \\
\hline \multicolumn{10}{|l|}{ Lazer } \\
\hline Sim & 19,59 & 6,60 & & 19,06 & 3,13 & & 3,64 & 2,15 & \\
\hline Não & 21,18 & 6,12 & 0,41 & 17,29 & 3,59 & 0,10 & 3,93 & 2,09 & 0,66 \\
\hline \multicolumn{10}{|l|}{ Ativ.Remunerada } \\
\hline Sim & 20,17 & 6,11 & & 18,07 & 3,58 & & 4,03 & 2,32 & \\
\hline Não & 21,31 & 6,73 & 0,56 & 17,75 & 3,45 & 0,77 & 3,44 & 1,59 & 0,36 \\
\hline
\end{tabular}

Fonte: Dados da pesquisa, 2008.

Nota: * Correlação significativa ao nível de 5\%

Na dimensão Exaustão, as mulheres apresentaram maior tendência a desenvolver sensação de esgotamento físico e mental. Os sujeitos na faixa de 21 a 25 anos parecem ser mais afetados pela a exaustão do que os demais. Acredita-se que as pessoas mais jovens têm uma maior dificuldade de se adaptar e de lidar com situações estressantes, como a rotina do mestrado. As pessoas que possuem filhos parecem ter estratégias mais adequadas para lidar com situações de estresse do que as pessoas que não possuem.

Na dimensão Realização Pessoal, os sujeitos mais experientes, casados e do sexo masculino se disseram mais realizados em suas vidas pessoais e nos estudos.

Na dimensão Cinismo, as pessoas que vivem em união estável e que são viúvas (variável "outros") relataram apresentar maior dificuldade em relação ao Programa de Mestrado e, consequentemente, no seu empenho.

Já em relação às variáveis acadêmicas, os resultados apontam para associação entre as dimensões de exaustão e cinismo e a variável "satisfação com o curso" (tabela 5). 
Rayssa Soares de Souza, Renata Paula Costa Trigueiro,

Tatiane Nunes Viana de Almeida e José Arimátes De Oliveira

Tabela 4: Relação entre dimensões de burnout e variáveis acadêmicas

\begin{tabular}{|c|c|c|c|c|c|c|c|c|c|}
\hline \multirow{2}{*}{$\begin{array}{l}\text { Dimensões } \\
\text { Variáveis }\end{array}$} & \multicolumn{3}{|c|}{ Exaustão } & \multicolumn{3}{|c|}{ Realização Pessoal } & \multicolumn{3}{|c|}{ Cinismo } \\
\hline & $\mathbf{M}$ & DP & $p$ & $\mathbf{M}$ & DP & $p$ & $\mathbf{M}$ & DP & $p$ \\
\hline \multicolumn{10}{|l|}{ Turma } \\
\hline 29 (Entrada 2007) & 19,38 & 6,26 & & 18,12 & 3,65 & & 4,23 & 2,34 & \\
\hline 30 (Entrada 2008) & 22,21 & 6,11 & 0,138 & 17,74 & 3,36 & 0,724 & 3,26 & 1,59 & 0,127 \\
\hline \multicolumn{10}{|l|}{ Área Concentração } \\
\hline Gestão Organiz. & 20,42 & 6,30 & & 18,04 & 3,26 & & 3,65 & 1,62 & \\
\hline Políticas Públicas & 20,79 & 6,43 & 0,849 & 17,84 & 3,89 & 0,855 & 4,05 & 2,63 & 0,534 \\
\hline \multicolumn{10}{|l|}{ Matrícula } \\
\hline 1 disciplina & 18,10 & 4,95 & & 18,80 & 3,68 & & 3,70 & 1,77 & \\
\hline 2 disciplinas & 21,91 & 7,25 & & 17,27 & 4,03 & & 4,73 & 3,07 & \\
\hline 3 disciplinas & 18,13 & 6,10 & & 18,88 & 3,14 & & 3,25 & 1,49 & \\
\hline Mais de 4 disc. & 22,44 & 6,03 & 0,194 & 17,44 & 3,29 & 0,527 & 3,56 & 1,67 & 0,485 \\
\hline \multicolumn{10}{|l|}{ Satisfação/curso } \\
\hline Muito satisfeito(a) & 16,71 & 5,34 & & 19,86 & 3,29 & & 2,36 & 1,08 & \\
\hline Satisfeito(a) & 22,52 & 6,11 & & 16,90 & 3,21 & & 4,45 & 2,16 & \\
\hline Indiferente & 19,50 & 0,71 & $0,019 \%$ & 20,00 & 4,24 & 0,071 & 5,00 & 1,41 & $0,001 *$ \\
\hline
\end{tabular}

Fonte: Dados da pesquisa, 2008.

Nota: * Correlação significativa ao nível de 5\%

Os indivíduos que se dizem satisfeitos com o Programa apresentaram um maior sentimento de esgotamento físico e emocional, podendo sugerir que essa exaustão esteja relacionada ao nível de esforço desprendido e engajamento com a pós-graduação. Já os pesquisados que se mostraram indiferente em relação ao curso, se disseram também indiferentes em relação aos estudos.

\section{CONCLUSÃO}

A presente pesquisa foi realizada com o objetivo de investigar as dimensões da síndrome de burnout em estudantes de Pós-Graduação. Essa investigação foi feita através do MBI-SS, onde foram identificadas as três dimensões da síndrome (exaustão, cinismo e realização pessoal) e estabeleceramse as correlações estatísticas entre os fatores da síndrome e as variáveis sócio-demográficas e acadêmicas.

Através do cálculo da análise por componentes principais, constatou-se no presente estudo variáveis enquadradas diferentemente dos resultados encontrados por Schaufeli et al. (2002), o que demonstra que os princípios teóricos tem correspondência empírica. Salienta-se que apesar das variáveis sóciodemográficas terem apresentado relação significativas com as dimensões da síndrome de burnout, as variáveis acadêmicas - satisfação e indiferença com o curso - também aparecem relacionadas com as dimensões negativas da síndrome, o que sugere uma atenção especial por parte do Programa, já que, como considera Carlotto e Gobbi (1999), o burnout não é um problema do indivíduo, mas sim do seu contexto de trabalho. Neste caso, do contexto acadêmico.

A limitação do presente estudo consiste na dificuldade em classificar os sujeitos nos níveis de baixo, moderado e alto. Logo, o estudo aponta para a necessidade de aprofundamento dos resultados obtidos, uma vez que a literatura atual ainda é relativamente restrita sobre burnout com esta população. Assim, sugere-se a realização de novos estudos, com outras variáveis e categorias estudantis.

\section{REFERÊNCIAS}

BENEVIDES-PEREIRA, A. M. T. Burnout: quando o trabalho ameaça o bem-estar do trabalhador. São Paulo: Casa do Psicólogo, 2002.

BORGES, A. M. B.; CARLOTTO, M. S. Síndrome de burnout e fatores de estresse em estudantes de um curso técnico de enfermagem. In: Aletheia, n.19, p.45-56, jan./jun. 2004. 
CARLOTTO, M. S.; CÂMARA, S. G. Características psicométricas do Maslach Burnout Inventory - Student Survey (MBI-SS) em estudantes universitários brasileiros. In: Psico-USF, v. 11, n. 2, p. 167-173, jul./dez. 2006.

; GOBBI, M. D. Síndrome de burnout: um problema do indivíduo ou do seu contexto de trabalho? Alethéia, v. 10, p.103-104, 1999.

CORRAR, L. J.; PAULO, E.; DIAS FILHO, J. M. (Coords.). Análise multivariada: para os cursos de administração, ciências contábeis e economia. São Paulo: Atlas, 2007.

DUQUE, J. C.; BRONDANI, J. T.; LUNA, S. P. L. Estresse e pós-graduação em medicina veterinária. In: Revista Brasileira de Pós-Graduação, v. 2, n. 3, p. 134-148, mar. 2005.

FRANÇA, H. H. A síndrome de burnout. In: Revista Brasileira de Medicina, v. 44, p.197-199, 1987.

GIL, A. C. Métodos e técnicas de pesquisa social. São Paulo: Atlas, 1999.

HAIR JÚNIOR, J. F.; ANDERSON, R. E.; TATHAM, R. L.; BLACK, W. C. Análise multivariada de dados. 5.ed. Porto Alegre: Bookman, 2005.

LIPP, M. E. N. Manual do inventário de sintomas de stress para adultos de Lipp (ISSL). São Paulo: Casa do Psicólogo, 2000.

MASLACH, C. Maslach burnout inventory Manual. Palo Alto, CA: Consulting Psychologists Press, 1986. Entendendo o Burnout. In: ROSSI, A. M.; PERREWÉ, P. L.; SAUTER, S. L. Stress e Qualidade de vida no Trabalho: Perspectivas atuais da saúde ocupacional. São Paulo, Atlas, 2007.

; LEITER, M. P. Trabalho: fonte de prazer ou de desgaste. Campinas: Papirus,1997

MATTAR, F. N. Pesquisa de marketing. Ed. Compacta. São Paulo: Atlas, 1996.

SAMARA, B. S.; BARROS, J. C. Pesquisa de marketing: conceitos e metodologia. 3. ed. São Paulo: Prentice Hall, 2002.

SANTOS; A. F.; ALVES JÚNIOR, A. Estresse e estratégias de enfrentamento em mestrandos de ciências da saúde. In: Psicologia: Reflexão e Crítica, 20 (1), p. 104- 113, 2007.

SCHAUFELI, W. B.; MARTINEZ, I. M.; PINTO, A. M.; SALANOVA, M.; BAKKER, A. B. Burnout and engagement in university students. In: Journal of Cross-Cultural Psychology, v.33, n. 5, p. 464-481, set. 2002.

TAMAYO, M. R. Relação entre a síndrome do burnout e os valores organizacionais no pessoal de enfermagem de dois hospitais públicos. 1997. 102 f. Dissertação (Mestrado em Psicologia) Universidade de Brasília, Distrito Federal, 1997.

TRZESNIAK, P. Qualidade e produtividade nos programas de pós-graduação: a disciplina seminário de dissertação. In: Revista Brasileira de Pós-Graduação, v. 1, p.111-125, jul. 2004.

VOLTARELLI, J. C. Estresse e produtividade acadêmica [Editorial]. In: Revista Medicina Ribeirão Preto, 35(4), p. 451-454, 2002. 